The Astrophysical Journal, 566:1166-1177, 2002 February 20

(C) 2002. The American Astronomical Society. All rights reserved. Printed in U.S.A.

\title{
UNCERTAINTIES IN DIELECTRONIC RECOMBINATION RATE COEFFICIENTS: EFFECTS ON SOLAR AND STELLAR UPPER ATMOSPHERE ABUNDANCE DETERMINATIONS
}

\author{
Daniel Wolf SAVIN \\ Columbia Astrophysics Laboratory, Columbia University, 550 West 120th Street, New York, NY 10027; savin@astro.columbia.edu \\ AND \\ J. MARTIN LAMING \\ E. O. Hulburt Center for Space Research, US Naval Research Laboratory, Code 7674L, Washington, DC 20375;jlaming@ssd5.nrl.navy.mil \\ Received 2001 February 1; accepted 2001 May 21
}

\begin{abstract}
We have investigated how the relative elemental abundances inferred from the solar upper atmosphere are affected by uncertainties in the dielectronic recombination (DR) rate coefficients used to analyze the spectra. We find that the inferred relative abundances can be up to a factor of $\approx 5$ smaller or $\approx 1.6$ larger than those inferred using the currently recommended DR rate coefficients. We have also found a plausible set of variations to the DR rate coefficients that improve the inferred (and expected) isothermal nature of solar coronal observations at heights of $\gtrsim 50^{\prime \prime}$ off the solar limb. Our results can be used to help prioritize the enormous amount of DR data needed for modeling solar and stellar upper atmospheres. Based on the work here, our list of needed rate coefficients for DR onto specific isoelectronic sequences reads, in decreasing order of importance, as follows: O-like, C-like, Be-like, N-like, B-like, F-like, Li-like, He-like, and Ne-like. It is our hope that this work will help to motivate and prioritize future experimental and theoretical studies of DR.
\end{abstract}

Subject headings: atomic data - atomic processes — stars: abundances — Sun: corona -

Sun: transition region

\section{INTRODUCTION}

It is now generally accepted that the elemental composition of the solar wind is different from that of the photosphere. Three decades of research have shown that in the slow-speed solar wind (which is believed to originate from quiet coronal regions) the abundance ratio of low first ionization potential (FIP) elements (FIP $<10 \mathrm{eV}$ ) relative to higher FIP elements is larger than it is in the photosphere. The observed FIP enhancement is roughly a factor of 4 . With the advent of the Ulysses spacecraft in its polar orbit, it has become clear that in the fast solar wind (which emanates from coronal holes) the enhancement of the low-FIP elements is certainly less than 2 and is perhaps consistent with the solar photospheric abundance pattern (von Steiger, Geiss, \& Gloeckler 1997; Feldman \& Laming 2000).

Similar phenomena are observed in spectroscopic studies of the solar corona and transition region, which we will refer to as the solar upper atmosphere. Mapping the FIP enhancement factor from the solar wind, through the corona and transition region, and into the photosphere (where, by definition, the factor is 1) is an important area of research for understanding the formation of the solar wind. FIP factors for the solar wind are determined from in situ particle measurements and are believed to be robust. FIP factors for the solar corona and transition region are inferred from spectroscopic observations and are sensitive to a number of uncertainties. Here we will explore how uncertainties in the dielectronic recombination (DR) rate coefficients limit our ability to infer FIP factors in the solar upper atmosphere.

Of the many ionization and recombination rate coefficients that go into ionization balance calculations for solar and stellar coronae (i.e., electron-ionized plasmas), the hightemperature DR rate coefficients are believed to be the most uncertain (Arnaud \& Raymond 1992; Mazzotta et al. 1998).
Unlike most other atomic processes (e.g., ionization and excitation) in which the direct contribution dominates the process, DR is solely a resonant process. These resonances are doubly excited, intermediate states that are highly correlated, which makes the calculations theoretically and computationally challenging.

Especially important are the high-temperature DR rate coefficients for ions with partially filled L shells. The astrophysical implications of these uncertainties are poorly understood. Investigating these implications is important because DR is the dominant electron-ion recombination process for most ions in electron-ionized plasmas.

Typically, inferences of the FIP effect from the solar corona are made from spectra that exhibit lines from several charge states of the same element (see, e.g., Malinovsky \& Heroux 1973; Laming, Drake, \& Widing 1995; White et al. 2000). In such cases any uncertainties in the DR rate coefficients and the ionization balance calculations essentially cancel out. This is because an error that might increase the fraction of ions in a particular charge state does so at the expense of ionic fractions in neighboring charge states. If one observes a whole series of charge states to evaluate the FIP effect (e.g., Fe IX, X, XI, XII, XIII, XIV, XV, and XVI in the case of White et al. 2000), these problems are to a large extent obviated.

Similar procedures are difficult to follow at solar transition region temperatures, where fewer elements have substantial series of observable charge states. The situation is compounded by the temperature dependence of the emission measure, as discussed in $\S 3.2$, and by the trend in solar physics instrumentation toward spectrometers with ever more limited bandpass while spatial and spectral resolution and sensitivity improve. For example, the spectrometers on the Solar Orbiting Heliospheric Observatory (SOHO) such as the Coronal Diagnostic Spectrometer (CDS; Harrison et al. 
1995, 1997) and the Solar Ultraviolet Measurements of Emitted Radiation (SUMER; Wilhelm et al. 1995, 1997; Lemaire et al. 1997) must scan their detectors across the bandpass to build up a full spectrum. This limits their utility in observing time-varying plasmas. The Extreme Ultraviolet Imaging Spectrometer (Culhane et al. 2002) will use multilayer coated gratings, which will further restrict the bandpass. The proposed wavelength ranges, $\approx 170-210$ and $\approx 250-290 \AA$, will provide good coverage in strong lines of $\mathrm{Fe}$ IX-Fe XVI and Fe XXIV but are less satisfactory for ions of other elements. For these reasons, accurate DR rate coefficients for the few lines that will be studied in current and future solar satellite missions have become more essential than ever.

Here we investigate the extent to which FIP enhancement factors, inferred using individual line ratios, can be affected by uncertainties in the DR rate coefficients used in the data analysis. We hope to motivate further experimental and theoretical studies of DR. We begin by reviewing in $\S 2$ the status of the theoretical DR results currently used in ionization balance calculations. We focus primarily on the rate coefficients for DR onto the L-shell ions Ne VI-Ne VIII, $\mathrm{Mg}$ VI-Mg IX, Si VI-Si XII, and S IX-S x. Lines from these ions are commonly observed from solar and stellar coronae. In $\S 3$ we discuss how relative abundances are determined for solar and stellar upper atmospheres and the current observational status of relative abundance determinations. We describe in $\S 4$ the ionization balance calculations and how the calculations are sensitive to our estimated uncertainties in the DR rate coefficients, and we present some implications. We conclude in $\S 5$ by making some specific recommendations for future directions in theoretical and experimental studies of DR.

\section{DIELECTRONIC RECOMBINATION}

Dielectronic recombination (DR) is a two-step process. It begins when a free electron collisionally excites an ion and is simultaneously captured (i.e., dielectronic capture). Core excitations of the ion can be labeled $N l j \rightarrow N^{\prime} l^{\prime} j^{\prime}$. Here $N$ is the principal quantum number of the core electron, $l$ is its angular momentum, and $j$ is the total angular momentum of the core electron. In general, the most important DR channels are those via $\Delta N \equiv N^{\prime}-N=0$ or 1 core excitations. The incident electron is captured into some $n l^{\prime \prime}$ Rydberg level, forming any one of an infinite number of intermediate doubly excited states of the recombining ion. These states can either autoionize or radiatively stabilize, the latter of which completes the DR process. Energy conservation requires $E_{k}=\Delta E-E_{b}$, where $E_{k}$ is the kinetic energy of the incident electron, $\Delta E$ the excitation energy of the core electron, and $\mathrm{E}_{b}$ the binding energy released when the free electron is captured. $\Delta E$ and $E_{b}$ are quantized, making DR a resonant process. The DR rate coefficient represents the convolution of these resonances with a Maxwellian electron energy distribution.

Here we are interested in low electron density, zero-field DR rate coefficients. Ionization balance calculations generally assume that electron densities are low enough that collisional ionization of the weakly bound electron captured in the DR process is unimportant. These calculations also assume that any external electric and magnetic fields are too weak to affect the DR rate coefficient and that metastable populations of all ions are insignificant.

\subsection{Overview of Theory and Experiment}

Reliable calculations of DR are extremely challenging theoretically and computationally. In contrast to most other atomic processes (e.g., ionization and excitation), in which the direct contribution dominates the process, DR is solely a resonant process. These resonances are doubly excited, intermediate states that are highly correlated. These states greatly complicate calculations since an accurate treatment of electron correlation is required (Zong et al. 1997). Calculations also require accounting for an infinite number of states. This is clearly impossible using a finite basis expansion. Approximations must be made to make the calculations tractable (Hahn 1993).

Many different theoretical techniques have been used to calculate DR rate coefficients for plasma modeling. In the past, semiempirical expressions such as the Burgess (1965) formula along with modified versions by Burgess \& Tworkowski (1976) and Merts, Cowan, \& Magee (1976) were derived to calculate DR rate coefficients. More recently, a number of sophisticated theoretical approaches have been developed, among them configuration-averaging (Griffin, Pindzola, \& Bottcher 1985), single-configuration LS coupling (McLaughlin \& Hahn 1984), quantum defect (Bell \& Seaton 1985), intermediate coupling (Badnell \& Pindzola 1989b), nonrelativistic multiconfiguration Hartree-Fock (MCHF; Nilsen 1986; Schippers et al. 1998), semirelativistic multiconfiguration Breit-Pauli (MCBP; Badnell 1986), fully relativistic multiconfiguration Dirac-Fock (MCDF; Chen 1985), and relativistic many-body perturbation theory (RMBPT; Zong et al. 1997) methods. Other methods include the Hebrew University Lawrence Livermore Atomic Code (HULLAC), which uses a relativistic, multiconfiguration, parametric potential method (Mitnik et al. 1998 ), and $R$-matrix methods, where radiative recombination (RR) and DR are treated in a unified manner in the close-coupling approximation (Nahar \& Pradhan 1994; Robicheaux et al. 1995). However, the result of all these different theoretical techniques and required approximations is DR rate coefficients that often differ by factors of 2-4 or more (Arnaud \& Raymond 1992; Savin et al. 1997, 1999; Savin 1999, 2000).

Cosmic plasmas are most commonly modeled using the recommended DR rate coefficients of Aldrovandi \& Péquignot (1973), Shull \& van Steenberg (1982), Nussbaumer \& Storey $(1983,1984,1986,1987)$, Arnaud \& Rothenflug (1985), Landini \& Monsignori Fossi (1991), Arnaud \& Raymond (1992), and Mazzotta et al. (1998). Essentially, none of these DR rate coefficients have been calculated using state-of-the-art techniques (i.e., MCHF, MCBP, MCDF, RMBPT, HULLAC, or $R$-matrix techniques that include the spin-orbit interaction). The vast majority of these have been calculated using single-configuration pure $L S$-coupling, semiempirical formulae, or isoelectronic interpolations.

It is important to carry out calculations using techniques more sophisticated than $L S$ coupling because $L S$-coupling calculations are known not to include all possible autoionization levels contributing to the DR process since they do not include the spin-orbit interaction. As a result, such calculations provide only a lower limit for the DR rate coefficient (Badnell 1988; Gorczyca \& Badnell 1996). For example, for lithium-like ions, Griffin et al. (1985) and Belić \& Pradhan (1987) discussed how $L S$ coupling accounts for 
only two-thirds of all possible $\Delta N=0$ recombining channels. Intermediate coupling calculations yield a DR rate coefficient for lithium-like C IV that is $50 \%$ larger than the $L S$-coupling result. Recent ion storage ring measurements and relativistic many-body perturbation calculations have verified the importance of these $L S$-forbidden autoionizing resonances (Mannervik et al. 1998). As for the various semiempirical formulae, Savin (1999) showed a priori that it is not possible to know which of the semiempirical formulae will yield a result close to the true DR rate coefficient and which will be off by a factor of 2 .

Because of the complexity of the theoretical descriptions for the DR process, it is almost impossible to say a priori which approximations in the calculations are justified and which are not. Laboratory measurements are needed to ensure that even state-of-the-art techniques produce reliable results. For example, initial Fe XVI MCBP results were a factor of $\approx 2$ larger than ion storage ring measurements (Linkemann et al. 1995). This discrepancy was later resolved (Gorczyca \& Badnell 1996), but without laboratory measurements, the error in the theory would probably have gone undiscovered for many years.

A related difficulty is that for many of the ions and DR resonances important in electron-ionized cosmic plasmas, there are very few experimental techniques capable of carrying out measurements at the accuracy needed to provide benchmarks useful for the theorists. Tokamak and theta pinch measurements suffer from factor of 2 uncertainties (Griem 1988). Crossed electron-ion beam techniques (Müller et al. 1987; Savin et al. 1996) have been limited in the energy range accessible as well as by low signal rates. Single-passes, merged electron-ion beam techniques (Dittner et al. 1987; Andersen, Bolko, \& Kvistgaard 1990; Andersen et al. 1992; Schennach et al. 1994) can cover a wider energy range but also have low signal rates. Because of a combination of poor statistics and poorly controlled external electric and magnetic fields, it is difficult to use most of these crossed- and merged-beam measurements to benchmark zero-field DR calculations. Using either technique, it is also difficult to determine the ion beam metastable population. Hence, these techniques cannot be used to carry out absolute measurements for many ions with partially filled shells (Badnell et al. 1991).

The two state-of-the-art experimental techniques for studying DR are electron beam ion traps (EBITs) and heavy-ion storage rings with a merged electron-ion beam interaction region. Both techniques store the ions long enough for all metastable states to decay radiatively to the ground state. Storage ring techniques measure absolute DR resonance strengths. EBITs produce relative resonance strength measurements, which can then be normalized to RR or electron impact excitation theory.

Until recently, EBIT measurements were essentially limited to studying only closed-shell systems (Beiersdorfer et al. 1992; Smith et al. 2000). This has changed with the recent studies of DR onto Fe XXI-Fe XXIV (Gu et al. 1999; $\mathrm{Gu}$ 2000). EBITs, however, are still not capable of reliably studying DR for collision energies $\lesssim 670 \mathrm{eV}$ (Wargelin, Kahn, \& Beierdorfer 2001). These are the dominant DR channels for most ions of second and third row elements, especially for ions with partially filled shells. Work is underway to overcome this limitation.

The majority of the storage ring measurements for astrophysically important ions have been carried out using the test storage ring (TSR) and CRYRING (Müller 1995). Measurements of DR resonance strengths and energies have been reported using TSR from 0 to $\approx 2200 \mathrm{eV}$ (Kenntner et al. 1995; Linkemann et al. 1995) and CRYRING from 0 to $\approx 50 \mathrm{eV}$ (DeWitt et al. 1995, 1996). Storage rings are the optimal technique for studying DR resonances at energies not accessible to EBITs. This makes storage rings particularly well suited for studying DR for ions of second and third row elements. The external fields in the merged-beam section are also extremely well controlled, which allows reliable, essentially zero-field DR measurements to be carried out (Savin et al. 2000). One limitation, though, is that ions with a charge-to-mass ratio $q / m \lesssim 0.1$ cannot be accelerated in CRYRING and TSR to a velocity at which electron capture from the rest gas in the ring becomes negligible. The resulting poor signal-to-noise ratio prevents measurements of DR for such ions (Schippers et al. 1998; Wolf 1999).

To date, EBIT and storage ring DR measurements have been limited in the isoelectronic sequences studied and the energy ranges covered. Few data sets exist that are comprehensive enough to benchmark high-temperature DR theory, and for those ions studied over a wide enough energy range, the benchmarked theory has not then been used to calculate the DR rate coefficients for all astrophysically important ions isoelectronic to the measured ion. As a result, only a very small fraction of the DR rate coefficients used for modeling electron-ionized cosmic plasmas have come from benchmarked state-of-the-art theory.

\subsection{Uncertainties in the Recommended DR Rate Coefficients}

Here we are interested in how uncertainties in the published DR rate coefficients affect the ionization balance calculations used to analyze solar and stellar spectra. The ions of interest are listed in $\S 1$. However, the calculated abundance for a given ion is affected by the atomic rate coefficients for the ions one lower and higher in charge state (and less so by ions two or more lower and higher). To account for this, we extend the list of ions of interest by one charge state on both the low and high end for each element considered. Below we review and estimate uncertainties in the published high temperature rate coefficients for DR onto $\mathrm{Ne}$ V-Ne IX, Mg V-Mg X, Si V-Si XIII, and S VIII-S XI.

In the absence of measurements, the best way to estimate the uncertainty in the theoretical DR rate coefficients is to compare calculations along an isoelectronic sequence. This yields a conservative estimate. Savin et al. (1999) showed that published rate coefficients do not necessarily give reliable upper and lower limits for the range in which the true DR rate coefficient lies. The true DR rate coefficient may lie outside of these limits. Hence, our approach here may, in fact, underestimate the uncertainties in the DR rate coefficients.

We compare the range of calculated DR rate coefficients to the recommended DR rate coefficients from Mazzotta et al. (1998) in order to estimate the uncertainties in the "stateof-the-art" for ionization balance calculations. Their DR rate coefficients are meant for use at temperatures between $10^{4}$ and $10^{9} \mathrm{~K}$. Wherever possible we also use laboratory measurements to benchmark theory. Because experimental data often do not exist for the ions we are interested in, we take into account measurements carried out on those isoelectronic ions that are closest in atomic number to the ions of interest. We also only take into account measurements of those resonances that are relevant to the DR rate coeffi- 
cients of interest here. In our discussion below, the predicted temperature of formation for an ion in an electronionized plasma is taken from the results of Mazzotta et al. Also, estimated uncertainties for DR rate coefficients onto a given ion are quoted for temperatures near where the ion peaks in fractional abundance in an electron-ionized plasma.

Based on the comparisons described below, for the ions of interest, we have put together a list of factors by which to scale the recommended DR rate coefficients of Mazzotta et al. (1998). These scale factors are listed in Table 1 . The $2^{6} \times 3^{3}$ possible combinations yield a total of 1728 sets of DR rate coefficients, which we will use in $\S 4$.

\subsubsection{Onto Helium-like Ne IX and Si XIII}

$L S$-coupling calculations were carried out by Jacobs et al. (1977b) for only Si XIII and by Romanik (1988). MCHF calculations were published by Nilsen (1986) and Karim \& Bhalla (1989). Chen (1986) has published MCDF rate coefficients. The results of Chen, Nilsen, Romanik, and Karim \& Bhalla are in excellent agreement, while the results of Jacob et al. are a factor of $\approx 2$ smaller. Mazzotta et al. (1998) use the rate coefficients of Chen.

EBIT and electron beam ion source studies of DR have been carried out for a number of helium-like ions. The most relevant measurements for our comparison here have been on Ne IX (Wargelin et al. 2000) and Ar XVII (Ali et al. 1991; Smith et al. 1996). There is an estimated total experimental uncertainty of $\approx 20 \%$ for these results. Overall there is good agreement between theory and experiment. Calculations using the technique of Karim \& Bhalla (1989) are in good agreement with the Ar XVII measurements of Ali et al. (1991). Calculations using the technique of Chen (1986) are in good agreement with the results on Ar XVII (Smith et al. 1996). For Ne Ix, for the sum of the measured Ne IX KLL resonances, the experiment lies a factor of 1.16 below calculations using the technique of Karim \& Bhalla (1989), a factor of 1.23 below calculations using the technique of Chen (1986), and a factor of 1.32 below calculations using the technique of Nilsen (1986).

Overall the calculations of Chen (1986) agree with laboratory results to within $\approx 20 \%$. We take this estimated $\pm 20 \%$ as the uncertainty in the recommended rate coefficients of Mazzotta et al. (1998) for DR onto Ne IX and Si XIII.

\subsubsection{Onto Lithium-like $\mathrm{Ne}$ VIII, $\mathrm{Mg} \mathrm{X}$, and $\mathrm{Si} \mathrm{XII}$}

$L S$-coupling DR calculations have been published by several different workers. Jacobs et al. (1977b, 1979) and

TABLE 1

SCALE Factors For the ReCommended DR Rate CoefFicients of MAZzotTA ET AL. (1998) ONTO SELECTED IONS OF $\mathrm{Ne}, \mathrm{Mg}, \mathrm{Si}$, AND S

\begin{tabular}{|c|c|c|c|c|c|}
\hline \multirow{2}{*}{$\begin{array}{c}\begin{array}{c}\text { Isoelectronic } \\
\text { Sequence }\end{array} \\
\mathrm{He} \ldots \ldots \ldots \ldots\end{array}$} & \multirow[b]{2}{*}{ Ne IX, Si XIII } & \multicolumn{4}{|c|}{ DR Scale Factors } \\
\hline & & $\ldots$ & 0.8 & 1.0 & 1.2 \\
\hline $\mathrm{Li} \ldots \ldots \ldots \ldots$ & Ne vIII, Mg x, Si XII & $\ldots$ & 0.8 & 1.0 & 1.2 \\
\hline $\mathrm{Be} \ldots \ldots \ldots \ldots$ & $\mathrm{Ne}$ VII, Mg IX, Si XI & $\ldots$ & $\cdots$ & 1.0 & 1.6 \\
\hline B $\ldots \ldots \ldots \ldots$ & Ne vI, Mg vIII, Si X & $\ldots$ & $\ldots$ & 1.0 & 1.7 \\
\hline $\mathrm{C} \ldots \ldots \ldots \ldots$ & Ne v, Mg vII, Si IX, S XI & 0.31 & 0.62 & 1.0 & $\ldots$ \\
\hline $\mathrm{N} \ldots \ldots \ldots \ldots$ & Mg vI, Si VIII, S x & $\ldots$ & 0.45 & 1.0 & $\ldots$ \\
\hline $\mathrm{O} \ldots \ldots \ldots \ldots$ & Mg v, Si vII, S IX & $\ldots$ & $\ldots$ & 1.0 & 3.6 \\
\hline $\mathrm{F} \ldots \ldots \ldots \ldots$ & Si VI, S VIII & $\ldots$ & $\ldots$ & 1.0 & 4.7 \\
\hline $\mathrm{Ne} \ldots \ldots \ldots \ldots$ & $\mathrm{Si} \mathrm{v}$ & $\ldots$ & 0.85 & 1.0 & $\ldots$ \\
\hline
\end{tabular}

Romanik (1988) published coefficients for Ne VIII, Mg X, and $\mathrm{Si}$ XII. Roszman (1987a) gave rate coefficients for Ne viII. MCDF results were published by Chen (1991) for Ne VIII and Si XII. For reasons discussed in $\S 2.1$, we do not use the published $L S$ results to estimate the uncertainty in the DR rate coefficients onto lithium-like ions.

Measurements have been carried out for a number of lithium-like ions. Storage ring measurements have been carried out on $\mathrm{Ne}$ VIII (Zong et al. 1998) and Si XII (Kenntner 1995; Kenntner et al. 1995; Bartsch et al. 1997). Uncertainties were typically $\approx 20 \%$.

At the temperature of peak formation, the dominant DR channel for lithium-like ions up to $\mathrm{Si}$ XII is via $\Delta N=0$ core excitations. For $\mathrm{Si}$ XII, the $\Delta N=0$ and $\Delta N=1$ $\left(N=2 \rightarrow N^{\prime}=3\right)$ contributions are comparable (Chen 1986). For $\Delta N=0$, DR onto Ne vIII MCBP theory was $\approx 20 \%$ below the experimental data. MCBP theory was $\approx 10 \%-20 \%$ below experimental data for $\Delta N=0$ DR onto Si XII. For Si XII, $\Delta N=1$ DR via $N=2 \rightarrow N^{\prime}=3$ core excitations, MCBP theory was larger than experimental data by $\approx 10 \%-20 \%$ for the $1 s^{2} 3 l 3 l$ resonances. For the $1 s^{2} 3 \ln l$ $(n \geq 4)$ resonances in Si XII, theory lies slightly below experiment. These comparisons suggest that the accuracy of the MCBP results is $\approx \pm 20 \%$.

None of the measured resonance strengths have been compared with results from the theoretical techniques used to calculate the published rate coefficients for Ne VIII, $\mathrm{Mg} \mathrm{X}$, and Si XII. This makes it difficult to use the measurements to infer an uncertainty in the recommended DR rate coefficients. We try to do this indirectly by comparing MCBP results with published MCDF results. The MCDF results of Chen (1991) agree to within $\approx 10 \%$ with the MCBP rate coefficients of Badnell \& Pindzola (1989b) for O VI and of N. R. Badnell \& M. O'Mullane (1999, private communication) for Ar xvI. Considering the comparisons between the MCBP and MCDF results and given the estimated uncertainty in the MCBP results, we therefore estimate that there may be an $\approx \pm 20 \%$ uncertainty in the MCDF rate coefficients of Chen (1991).

Mazzotta et al. (1998) use the rate coefficients of Chen (1991) and interpolate isoelectronically for those ions that Chen did not calculate. Chen did not present results for $T \lesssim 10^{5} \mathrm{~K}$. As a result, the fitted rate coefficients of Mazzotta et al. do not have the correct low-temperature behavior. This can readily be seen by plotting their recommended rate coefficient for $\mathrm{C}$ IV and comparing it with published $\mathrm{C}$ IV rate coefficients (Schippers et al. 2001). This error in the rate coefficients of Mazzotta et al. will affect ionization balance calculations for photoionized plasmas but is expected to have little effect on modeling electron-ionized plasmas. Given the estimated uncertainty in the results of Chen, we estimate that for Ne VIII, $\mathrm{Mg} \mathrm{X}$, and Si XII, there is an $\approx \pm 20 \%$ uncertainty in the relevant DR rate coefficients of Mazzotta et al.

\subsubsection{Onto Beryllium-like $\mathrm{Ne}$ VII, $\mathrm{Mg} \mathrm{IX}$, and $\mathrm{Si} \mathrm{XI}$}

$L S$-coupling rate coefficients were published for Ne vII, Mg IX, and Si XI by Jacobs et al. (1977b, 1979) and Romanik (1988). Near the temperatures of peak formation, their hightemperature results are in good agreement. MCBP results were presented by Badnell (1987) for $\mathrm{Ne}$ VII, $\mathrm{Mg}$ IX, and $\mathrm{Si}$ XI. At the temperature of peak formation, the rate coefficients of Jacobs et al. and Romanik are a factor of $\approx 1.4-1.6$ times larger than those of Badnell. 
Mazzotta et al. (1998) use the rate coefficients of Badnell (1987) and appear to interpolate along the isoelectronic sequence for those ions that Badnell did not calculate. Badnell does not present any results for $T<10^{5} \mathrm{~K}$. A comparison of the rate coefficients from Mazzotta et al. with those from Romanik (1988) shows that the former do not have the correct low-temperature behavior. This will be important in modeling the ionization structure of photoionized plasmas but is not an issue here.

In the absence of laboratory benchmarks, we use the various published DR calculations to provide upper and lower limits for the DR rate coefficients. As discussed above, this is a conservative estimate of the uncertainty. We estimate that for Ne VII, Mg IX, and Si XI, the uncertainty in the relevant DR rate coefficients of Mazzotta et al. (1998) is $+60 \%$ and $-0 \%$.

\subsubsection{Onto Boron-like $\mathrm{Ne}$ VI, $\mathrm{Mg}$ VIII, and $\mathrm{Si} \mathrm{x}$}

Jacobs et al. (1977b, 1979) reported $L S$-coupling DR rate coefficients for Ne VI, Mg VIII, and Si X. Nahar (1995) used $R$-matrix techniques to calculate $\mathrm{RR}+\mathrm{DR}$ rate coefficients in $L S$-coupling for $\mathrm{Ne}$ VI, $\mathrm{Mg}$ VIII, and $\mathrm{Si}$ x. Mazzotta et al. (1998) recommends DR rate coefficients based on the calculations of Nahar after apparently subtracting the theoretical RR rate coefficient from her results.

Given the paucity of theoretical calculations and appropriate laboratory measurements, it is difficult to estimate the uncertainty in the theoretical DR rate coefficients for the ions of interest. We attempt to do this indirectly by comparing the results for DR onto the isoelectronic ions $\mathrm{C}$ II, N III, and $\mathrm{O}$ IV for which a number of different calculations exist. $L S$-coupling rate coefficients were reported by Jacobs et al. (1978) and Ramadan \& Hahn (1989). Fits to the results of Jacobs et al. (1978) were reported by Shull \& van Steenberg (1982). $R$-matrix results using $L S$-coupling were presented by Nahar (1995). Badnell \& Pindzola (1989a) carried out MCBP calculations, and Safronova \& Kato (1998) carried out MCHF calculations. The various theoretical techniques used have not yet converged to the same rate coefficients. Mazzotta et al. (1998) recommend the rate coefficients of Nahar (1995). At the temperatures of peak formation for $\mathrm{C}$ II, $\mathrm{N}$ III, and $\mathrm{O}$ IV, the other theoretical rate coefficients are a factor of $\approx 1.0-1.7$ larger than the recommended rate coefficients. We estimate that for Ne VI, $\mathrm{Mg}$ VIII, and Si $\mathrm{x}$, the uncertainty in the relevant DR rate coefficients of Mazzotta et al. is $+70 \%$ and $-0 \%$.

\subsubsection{Onto Carbon-like $\mathrm{Ne} \mathrm{v}, \mathrm{Mg}$ VII, Si IX, and S XI}

There have been very few calculations of hightemperature DR for the ions of interest here. $L S$-coupling results have been presented for Ne V, Mg VII, Si IX, and S XI by Jacobs et al. (1977b, 1979). Mazzotta et al. (1998) use the rate coefficients of Jacobs et al. scaled up by a factor of $\approx 1.6$. This factor was apparently derived by scaling the isoelectronic Fe XXI results of Jacobs et al. (1977a), calculated using the same technique as Jacobs et al. (1977b, 1979), to the recommended DR rate coefficient of Arnaud \& Raymond (1992).

Considering how few relevant calculations exist, it is difficult to estimate the uncertainty in the DR rate coefficients for the ions of interest here. We attempt to do this indirectly using theoretical results for high-temperature DR onto N II and O III. Jacobs et al. (1978) presented $L S$-coupling results for N II and O III that were fitted by Shull \& van Steenberg
(1982). $R$-matrix results in $L S$-coupling were calculated by Nahar \& Pradhan (1997) for N II and by Nahar (1999) for $\mathrm{O}$ III. MCBP and $L S$ rate coefficients were calculated by Badnell \& Pindzola (1989b) and Roszman (1989), respectively, for O III. Note that there is an apparent error in Table 1 of Roszman (1989). We had to reduce the DR rate coefficients by an order of magnitude in order for the tabulated rate coefficients to match those shown in Figures 1 and 2 of his paper. Near the high-temperature DR peak for $\mathrm{N}$ II, the rate coefficient of Nahar \& Pradhan (1997) is a factor of $\approx 2.2$ smaller than that of Jacobs et al. For O III near this peak, the rate coefficient of Roszman is a factor of $\approx 1.2$ times larger than that of Jacobs et al. The rate coefficients of Badnell \& Pindzola and Nahar \& Pradhan are $\approx 1.25$ and $\approx 1.7$ smaller, respectively, than the results of Jacobs et al.

For DR onto Ne V, Mg VII, Si IX, and S XI, we use the recommended rate coefficients of Mazzotta et al. (1998). A reduction in these rate coefficients by $38 \%$ brings their results into agreement with the original results of Jacobs et al. Considering the comparison of the various theoretical DR rate coefficients of $\mathrm{N}$ II and $\mathrm{O}$ III, a reduction in the rate coefficients of Mazzotta et al. by $69 \%$ represents our estimated lower limit to the uncertainty in their recommended DR rate coefficients.

\subsubsection{Onto Nitrogen-like $\mathrm{Mg}$ VI, $\mathrm{Si}$ VIII, and $\mathrm{S} \mathrm{x}$}

We are aware only of the $L S$-coupling calculations by Jacobs et al. (1977b, 1979) for Mg vI, Si vIII, and S x. Mazzotta et al. (1998) use the rate coefficients of Jacobs et al. For S x, Mazzotta et al. do not include the low-temperature results of Jacobs et al. This is not an issue here but may be important in photoionized plasmas.

The paucity of calculations for DR onto nitrogen-like ions makes it difficult to estimate the uncertainty in the published theoretical rate coefficients. We attempt to do this indirectly using published rate coefficients for DR onto the isoelectronic O II. High-temperature DR rate coefficients have been calculated using $L S$-coupling by Jacobs et al. (1978) and Terao et al. (1991). Fits to the results of Jacobs et al. were published by Shull \& van Steenberg (1982). Nahar (1999) published $R$-matrix rate coefficients (e.g., $\mathrm{RR}+\mathrm{DR}$ ) using $L S$-coupling. Intermediate coupling calculations have been given by Badnell \& Pindzola (1989b), which were later improved on by Badnell (1992).

The results of Jacobs et al. (1978) and Badnell \& Pindzola (1989b) are in good agreement. However, Badnell (1992) recalculated the DR rate coefficient taking into account correlation between the $n=2$ and $n=3$ shells. His new rate coefficient is a factor of $\approx 1.6$ times smaller than that of Jacobs et al. (1978) and Badnell \& Pindzola (1989a). Terao et al. (1991) revised their published rate coefficients upward by $\approx 20 \%$ (Badnell 1992). This revised result is a factor of $\approx 2.2$ smaller than the results of Jacobs et al. (1978). To determine the DR rate coefficient of Nahar (1999), we subtracted out the estimated RR rate coefficient. By extrapolating her recombination rate coefficient at low temperatures, where DR is unimportant, we estimated the $\mathrm{RR}$ rate coefficient at the relevant high temperatures. The resulting DR rate coefficient lies a factor of $\approx 2.2$ below that of Jacobs et al. Taking into account these comparisons, we estimate the uncertainty in the relevant rate coefficients of Mazzotta et al. for DR onto Mg VI, Si vIII, and S x to be $+0 \%$ and $-55 \%$. 


\subsubsection{Onto Oxygen-like $\mathrm{Mg}$ v, Si VII, and $\mathrm{S}$ IX}

Jacobs et al. (1977b, 1979) have published $L S$ rate coefficients for $\mathrm{Mg}$ v, Si vII, and S IX. These rate coefficients were fitted by Shull \& van Steenberg (1982). Mazzotta et al. (1998) use these results. We estimate the uncertainties in the DR rate coefficients for these ions by comparing the rate coefficients for DR onto the isoelectronic Fe XIX for which a number of different calculations exist. Jacobs et al. (1977a) and Roszman (1987c) published $L S$-coupling results. Jacobs et al. (1977a) used the same technique as Jacobs et al. (1977b, 1979). Their results were fitted by Shull \& van Steenberg (1982) for use in ionization balance calculations. Hartree-Fock calculations with relativistic corrections, using the code of Cowan (1981), were reported by Dasgupta \& Whitney (1994).

In making our comparisons between the different calculations, we need to take into account the relative importance of the different DR channels for the ions of interest. For Fe XIX, near the temperature of peak formation the $\Delta N=1\left(N=2 \rightarrow N^{\prime}=3\right)$ channel appears to dominate the DR process (Roszman 1987c; Dasgupta \& Whitney 1994). It is unclear how this extrapolates to the less highly charged Mg V, Si vII, and S IX.

For Fe XIX at the temperature of peak formation, the rate coefficients of Roszman (1987c) and Dasgupta \& Whitney (1994) are factors of approximately 2.7 and 3.6 times larger, respectively, than the result of Jacobs et al. (1977a). Mazzotta et al. use the rates of Jacobs et al. (1977b, 1979) for Mg V, Si VII, and S IX. Based on our comparison for Fe XIX, we estimate the uncertainty in the high-temperature rate coefficients for DR onto these ions to be $+260 \%$ and $-0 \%$.

\subsubsection{Onto Fluorinelike Si VI and S VIII}

Jacobs et al. (1977b, 1979) carried out $L S$-coupling calculations for Si VI and S VIII. We are unaware of any other calculations for these ions. Shull \& van Steenberg (1982) fit the data of Jacobs et al. Mazzotta et al. (1998) use these fits.

We can estimate the important DR channels in Si VI and $S$ VIII and the uncertainties in the relevant DR rate coefficients using the theoretical results for the isoelectronic ions Fe XVIII. Jacobs et al. (1977a), using the same techniques as Jacobs et al. (1977b, 1979), have published an LS rate coefficient. Shull \& van Steenberg (1982) presented a fit to this rate coefficient for use in plasma modeling. Roszman (1987b) calculated an $L S$ rate coefficient. Dasgupta \& Whitney (1990) published Hartree-Fock calculations with relativistic corrections using the code of Cowan (1981). MCDF results were reported by Chen (1988a). At the temperatures of peak formation for Fe XVIII, the $\Delta N=1$ $\left(N=2 \rightarrow N^{\prime}=3\right)$ channel dominates the DR process. It is likely that the same situation exists for Si vi and S vIII. At the temperature of peak formation for Fe XVIII, the rate coefficients of Roszman (1987b), Chen (1988a), and Dasgupta \& Whitney (1990) lie factors of approximately 3.5, 4.2, and 4.7, respectively, above the results of Jacobs et al. (1977a). Here, for Si VI and S VIII, we use the recommended high-temperature DR rate coefficients of Mazzotta et al. (1998) and estimate the uncertainty to be $+370 \%$ and $-0 \%$.

\subsubsection{Onto Neon-like Si V}

Jacobs et al. (1977b) and Romanik (1988) have calculated DR rate coefficients using $L S$-coupling for $\mathrm{Si}$ v. At the peak in the DR rate coefficient, the results of Jacobs et al. lie a factor of $\approx 1.9$ below those of Romanik. Mazzotta et al. (1998) use the results of Romanik. We use the theoretical rate coefficients for DR onto Ar IX to estimate the uncertainty in the recommended $\mathrm{Si} v$ data. $L S$ rate coefficients were given by Romanik. Chen (1986) published MCDF rate coefficients. HULLAC results were given by Fournier, Cohen, \& Goldstein (1997).

The results of Romanik (1988) and Fournier et al. (1997) are in excellent agreement. The calculations of Chen (1986) lie $\approx 15 \%$ below those of Romanik and Fournier et al. The close agreement between these three different calculations and the large difference between the results of Jacobs et al. (1977b) and Romanik for Si v strongly suggest an error in the reported results of Jacobs et al. This is supported by a comparison of rate coefficients for DR onto Fe XVII by Arnaud \& Raymond (1992). They found that the results of Chen and Romanik were in good agreement but that the results of Jacobs et al. (1977a), calculated using the same techniques as Jacobs et al. (1977b), were a factor of $\approx 4.7$ times smaller. Considering these various comparisons, we use the recommended rate coefficient of Mazzotta et al. (1998) for $\mathrm{Si} \mathrm{v}$ and estimate the uncertainty to be $+0 \%$ and $-15 \%$.

\section{SOLAR AND STELLAR UPPER ATMOSPHERE ABUNDANCE OBSERVATIONS}

\subsection{Determining Relative Abundances}

Spectral line ratios can be used to determine relative abundances in cosmic plasmas. Photons are emitted in a spectral line at a rate per second per steradian given by

$$
R=\frac{A_{j i}}{8 \pi} \int_{\Delta V} \frac{n_{j}}{n_{q}} \frac{n_{q}}{n_{A}} \frac{n_{A}}{n_{\mathrm{H}}} \frac{n_{\mathrm{H}}}{n_{e}} n_{e} d V,
$$

where $R$ is the rate, $A_{j i}$ the radiative decay rate from excited ionic level $j$ to $i, n_{j}$ the number density of level $j, n_{q}$ the number density for the $q$-times charged ion in question, $n_{A}$ the number density for the element from which this ion is formed, $n_{\mathrm{H}}$ the hydrogen number density, $n_{e}$ the electron number density, and $\Delta V$ the emitting volume. The radiation is emitted into $4 \pi$ sr. For full-disk spectra, it is usual to include a factor of $\frac{1}{2}$ to account for photons emitted toward the solar disk and absorbed by the photosphere. At low densities (i.e., in coronal plasma), we can write $n_{j} / n_{q}=$ $C_{j} n_{e} / A_{j i}$, where $C_{j}$ is the total excitation rate coefficient of the excited level $j$ by electron collisions and we have assumed the branching ratio for the $j \rightarrow i$ radiative transition to be 1 . For the transitions of interest in the solar FIP diagnostics under consideration here (i.e., $n=2 \rightarrow n=2$ transitions in $L$-shell ions), excitation rate coefficients due to other processes are negligible compared to those from electron impact excitation. Defining $f_{q}=n_{q} / n_{A}$ and changing the integration variable from volume to temperature gives

$$
R=\frac{1}{8 \pi} \frac{n_{A}}{n_{\mathrm{H}}} \frac{n_{\mathrm{H}}}{n_{e}} \int_{\Delta T} C_{j} f_{q} n_{e}^{2} \frac{d V}{d T} d T,
$$

where parameters not dependent on the electron temperature have been taken outside the integral. The quantity $n_{e}^{2} d V / d T$ is the differential emission measure (DEM, also sometimes defined as $n_{e}^{2} d V / d \log T$ ). The usual procedure in determining the DEM is to assume some functional form (usually DEM $\propto T^{a}$ ), evaluate $R$ for all lines, compare these values with observations, and iterate on the DEM distribu- 
tion until satisfactory agreement is obtained. This can be done for all the lines of one particular element (usually Fe). Then lines from other elements can be analyzed the same way to determine relative element abundances. However, in this procedure the role of uncertainties in particular atomic rate coefficients becomes obscured by the quantity of data and number of iterations required.

A second technique for determining relative abundances is to use a line from an ion of a specific element and another line from an ion of a different element. This is the technique that will most likely be used to infer relative abundances from future solar satellite missions. The lines are chosen so that the respective values of $C_{j} f_{q}$, to a first approximation, have similar dependences on temperature, differing only by a multiplicative constant. Using equation (2) to take the ratio of the two lines, it is easy to show that the ratio of the two integrals reduces to this constant, which is determined from atomic parameters, times the relative abundances. In this approximation the shape of the DEM has no affect on the results. This technique has the advantage of allowing one to study how uncertainties in atomic physics affect the inferred relative abundances.

\subsection{Solar Disk Observations}

The first systematic analysis of coronal abundances over a wide temperature region using high-quality spectral data was probably that of Laming et al. (1995), who used a variant of the first DEM method described above. They analyzed the full-disk solar corona spectrum of Malinovsky $\&$ Heroux (1973) and covered a range of $\log T=5.5-6.5$. They found an abundance pattern consistent with that of the slow-speed solar wind, at least for solar coronal plasma with an electron temperature $\log T>5.8$ (i.e., $T>9 \times 10^{5}$ K). More recent studies with lines from ions formed at similar high temperatures corroborate this result (Laming et al. 1999; White et al. 2000), but in what was a surprise at the time, they found essentially no FIP enhancement in transition region plasma at $\log T<5$.8. A similar result was found earlier by Noci et al. (1988), who used data from the Skylab S-055 extreme-spectroheliometer.

Interpreting spectra from these cooler temperatures poses a number of problems. This may be in part due to complications in the solar physics at these temperatures (Feldman \& Laming 1994) or to problems in the interpretation of the data. At transition region temperatures, the solar emission measure $\mathrm{EM}=n_{e}^{2} V(T)$ is an increasing function of $T$. The "canonical" quiet-Sun behavior is EM $\propto T^{1.5}$ (Jordan 1980), although in certain solar features power laws of $T^{4}$ or higher may be appropriate (Cargill 1994; Cargill \& Klimchuk 1997). This is quite different from the situation at $\log T>6$, where in the quiet Sun the emission measure is in general much less steep and may even be flat in certain temperature regimes (see, e.g., Laming et al. 1995). One consequence of the EM temperature dependence is that in observations of ions formed at $\log T>5.8$, where the emission measure is not significantly temperature-dependent, the detected line emission comes primarily from regions near the temperatures of peak fractional abundance for the observed ions. However, this is not the case for ions with formation temperatures of $\log T<5.8$. For these ions, the steeply rising EM skews the temperature range over which emission from the ions is sampled to temperatures well above those where the ions peak in fractional abundance. Under these conditions uncertainties in the atomic data used to calculate the ionization balance can significantly affect the inferred properties of the observed plasma. In addition, at temperatures significantly above or below those where an ion is formed in ionization equilibrium, we find that the fractional ion abundances calculated by balancing the electron-ion ionization and recombination rate coefficients are extremely sensitive to errors in the atomic data.

Much of the work prior to that of Laming et al. (1995) had clearly observed FIP effects at $\log T<5.8$, principally using the $\mathrm{Mg}$ VI/ $\mathrm{Ne}$ VI and $\mathrm{Ca} \mathrm{IX} / \mathrm{Ne}$ VII intensity ratios in the second, simpler method outlined in $\S 3.1$ for determining relative abundances (Feldman \& Widing 1990, 1993; Widing \& Feldman 1989, 1992, 1993). One reason for the apparent disagreement between these studies and that of Laming et al. (1995) is probably that while Laming et al. studied a full-disk-integrated spectrum, the prior work had concentrated on discrete solar features observed by the Skylab SO-82A spectroheliograph. Specifically, the Skylab investigations had concerned a coronal polar plume (Widing \& Feldman 1992), an impulsive flare (Feldman \& Widing 1990), an open-field active region (Widing \& Feldman 1993), or a variety of such features (Widing \& Feldman 1989).

Interestingly, the study of a coronal hole using an $\mathrm{Mg} \mathrm{VI} /$ Ne vi line ratio (Feldman \& Widing 1993) yielded an apparent FIP enhancement of about $2-2.5$ at $\log T \approx 5.6$. This is slightly higher than the findings of Laming et al. (1995) for the full-disk Sun in this lower temperature range. However, given the estimated uncertainties of $50 \%$ in Feldman \& Widing (1993) and Laming et al. (1995) in this temperature range and the different methods of analysis, there is a suggestion that these results are in agreement. The FIP enhancements of Feldman \& Widing (1993) are also similar to or slightly higher than those observed in the fast-speed solar wind, which is believed to emanate from coronal holes. The results of Feldman \& Widing (1993) and Laming et al. (1995) support the hypothesis that the FIP factor decreases as one moves inward from the solar corona to the transition region.

A considerable amount of attention has been given to the open-field active region reported by Widing \& Feldman (1993) and the coronal polar plume reported by Widing \& Feldman (1992). This is due to the observed FIP enhancements being greater than an order of magnitude. SOHO observations of open-field active regions using CDS have found results (Young \& Mason 1997) similar to those of the active region of Widing \& Feldman (1993). SOHO observations have also been carried out of coronal polar plumes, principally using SUMER. Doschek et al. (1998) made observations of the $\mathrm{Si} / \mathrm{Ne}$ abundance ratio (as a proxy for the FIP effect) and found an FIP enhancement of only a factor of 2. Other SUMER observers find similar results (del Zanna \& Bromage 1999; Young, Kilmchuk, \& Mason 1999). So far, it has not been possible to reproduce the polar plume results of Widing \& Feldman (1992).

The emission measure in the open-field active region analyzed by Widing \& Feldman (1993) is relatively flat with temperature, and the inferred FIP enhancements of these features are believed to be reliable. However, the emission measure plotted by Widing \& Feldman (1992) for their observed coronal polar plume rises very steeply with temperature. Although the Mg vi and Ne vi ionization fractions match each other very well near their temperature of peak formation, at higher temperatures the $\mathrm{Mg}$ VI fraction 
becomes larger than that for Ne vI. This is illustrated in Figure 1, where the relative intensities are plotted for $\mathrm{Mg}$ vI $\lambda 1190.09$ and $\mathrm{Ne}$ vi $\lambda 558.59$ using the ionization balance of Arnaud \& Rothenflug (1985). The excitation rate coefficients are taken to vary as $\exp (-\Delta E / k T) / \sqrt{T}$, where $\Delta E$ is the excitation potential and $T$ is the electron temperature. Under conditions where the temperature structure of the plasma weights the emission from these lines so that the temperature region greater than $5 \times 10^{5}$ is dominant, apparent abundance enhancements of $\mathrm{Mg}$ over $\mathrm{Ne}$ may result simply because of the relative ionization fractions. Realistic calculations by Doschek \& Laming (2000) for the emission measure distributions found by Widing \& Feldman (1992) suggest that an extra factor of 2-3 in the intensity ratio, on top of the usual FIP effect, could result from this effect. Thus, if the true FIP effect is a factor of 2-4, an apparent abundance enhancement of an order of magnitude or more could result.

\subsection{Stellar Observations}

All observations of stellar coronae are necessarily diskintegrated. In general, in spectra of stellar coronae, individual line ratios for FIP effect diagnostics are not as readily available as they are in solar spectra. Most abundance results have been derived using these spectra from emission measure plots and related techniques, where the effect of uncertainties in DR is much less transparent. However, two papers that do use specific line ratios are Drake et al. (1995) on Procyon and Drake, Laming, \& Widing (1997) on $\alpha$ Cen. These sources are perhaps the two best-observed stellar coronae with the Extreme Ultraviolet Explorer (EUVE).

In general, analyses on $E U V E$ spectra of solar-like stars have found coronal abundance anomalies similar to the solar FIP effect. Only Procyon appears to have a photospheric abundance corona. Coronal abundances in active binary stars do not show such effects but hint at a trend of decreasing coronal metallicity with increasing activity. These and other results prior to the launch of the Chandra

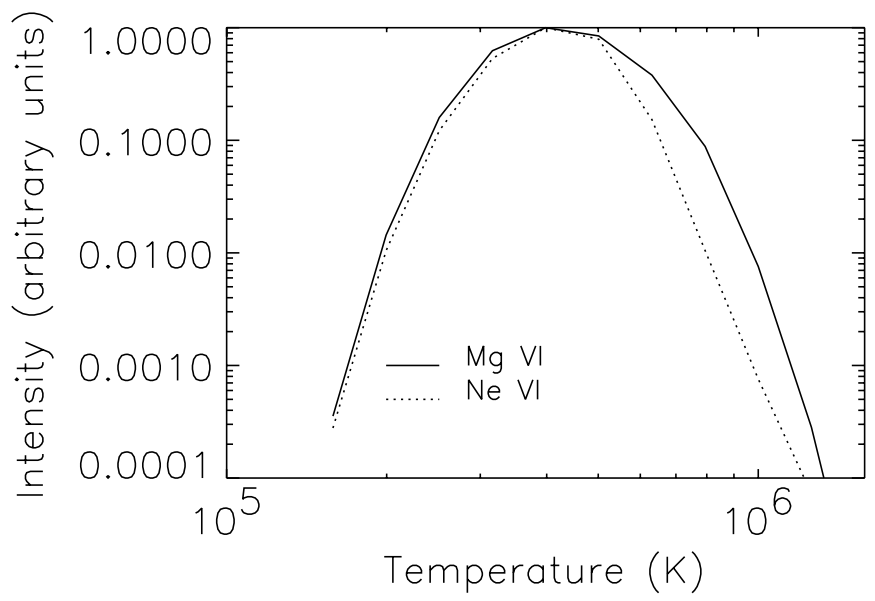

FIG. 1.- Intensity curves for the emissivity (photons per second per unit electron density per ion) times the ionization fraction of $\mathrm{Mg}$ VI $\lambda 1190.09$ and $\mathrm{Ne}$ VI 2558.59 . Both curves have been normalized to their maximum values. The correspondence between the two is almost exact for $T \leq 5 \times 10^{5} \mathrm{~K}$. At higher temperatures, the $\mathrm{Mg}$ vi line is stronger. A steeply rising emission measure may weight emission from this temperature region enough to produce large apparent $\mathrm{Mg} / \mathrm{Ne}$ abundance ratios if this difference between the two curves is not accounted for during data analysis. and $X M M$-Newton satellites are reviewed by Feldman \& Laming (2000). With the advent of high-resolution stellar X-ray spectroscopy with Chandra and XMM-Newton, the field of stellar coronae and their abundances is set to become a rich area of research. Already new results have raised the possibility of yet more varied coronal abundance patterns, e.g., the inverse FIP effect (Brinkmann et al. 2001) or enhanced Ne and $\mathrm{Ar}$ (Drake et al. 2001) in the coronae of HR 1099.

\section{EFFECTS OF DR UNCERTAINTIES ON RELATIVE ABUNDANCE DETERMINATIONS}

Our work focuses on studying particular line ratios because of their importance for determining relative abundance ratios using data collected from past, current, and future solar satellite missions. These line ratios also offer a relatively transparent insight into the effect of uncertainties in the DR rate coefficients on abundance measurements. SOHO studies have been carried out using $\mathrm{Mg} \mathrm{VI} / \mathrm{Ne} \mathrm{VI}$, $\mathrm{Mg}$ VII/Ne VII, $\mathrm{Mg}$ IX/S Ix, Mg IX/S x, Si Ix/S IX, and Si IX/ $\mathrm{S} \times$ line ratios. Wavelengths for the observed lines are given in detail in Table 3. An additional abundance ratio that is of interest is $\mathrm{Si} \mathrm{x} / \mathrm{S} \mathrm{x}$, derived from $\mathrm{Si} \mathrm{x} \lambda 258.40$ and $\lambda 261.27$ and $S x$ transitions at 259.50 and $264.24 \AA$, which have been used in EUVE abundance studies on the coronae of $\alpha$ Cen (Drake et al. 1997). We consider all of the seven line ratios listed above in our discussion below.

\subsection{Ionization Balance Calculations} by

The ionization fraction $f_{q}$ of the ion with charge $q$ is given

$$
\begin{aligned}
\frac{d f_{q}}{d t}= & n_{e}\left[C_{\mathrm{ion}, q-1} f_{q-1}-C_{\mathrm{ion}, q} f_{q}\right. \\
& \left.+\left(C_{\mathrm{RR}, q+1}+C_{\mathrm{DR}, q+1}\right) f_{q+1}-\left(C_{\mathrm{RR}, q}+C_{\mathrm{DR}, q}\right) f_{q}\right],
\end{aligned}
$$

where $C_{\mathrm{ion}, q}, C_{\mathrm{RR}, q}$, and $C_{\mathrm{DR}, q}$ are the rate coefficients for electron impact ionization, $R R$, and DR, respectively, out of the charge state $q$. For the coronal plasmas under consideration here, three-body recombination can be safely neglected. In ionization equilibrium, $d f_{q} / d t=0$, which gives $Z$-independent linear equations for the $Z+1$ charge states. The constraint that all charge state fractions must add up to unity supplies the final equation, allowing us to solve the $Z+1$ linear equations by LU decomposition (see Press et al. 1992 for details) of the matrix formed by the right-hand side of equation (3). This set of linear equations is solved repeatedly with rate coefficients appropriate to different electron temperatures to find the temperature dependence of the different charge state fractions. Initially, the ionization and RR and DR rate coefficients are taken from the same sources as used by Mazzotta et al. (1998).

\subsection{Relative Abundances}

Using equation (2), the relative abundances for two elements, $A 1$ and $A 2$, are given by

$$
\frac{n_{A 1}}{n_{A 2}}=\frac{R_{A 1}}{R_{A 2}} \frac{\int_{\Delta T} C_{j 2} f_{q}^{\mathrm{A} 2} n_{e}^{2}(d V / d T) d T}{\int_{\Delta T} C_{j 1} f_{q}^{\mathrm{A} 1} n_{e}^{2}(d V / d T) d T} .
$$

This equation is first evaluated with ionization balance calculations that use the unscaled DR rate coefficients of Mazzotta et al. (1998). To investigate the effects that the various sets of DR rate coefficients have on the inferred value of 
$n_{A 1} / n_{A 2}$, we define the quantity

$$
\begin{aligned}
S= & {\left[\frac{\int_{\Delta T} C_{j 2} f_{q}^{\mathrm{A} 2} n_{e}^{2}(d V / d T) d T}{\int_{\Delta T} C_{j 1} f_{q}^{\mathrm{A} 1} n_{e}^{2}(d V / d T) d T}\right]_{\text {new }} / } \\
& {\left[\frac{\int_{\Delta T} C_{j 2} f_{q}^{\mathrm{A} 2} n_{e}^{2}(d V / d T) d T}{\int_{\Delta T} C_{j 1} f_{q}^{\mathrm{A} 1} n_{e}^{2}(d V / d T) d T}\right]_{\mathrm{old}} }
\end{aligned}
$$

where the subscripts "old" and "new" refer to ionization balance calculations carried out using the unscaled and scaled DR rate coefficients of Mazzotta et al. (1998), respectively. Multiplying the right-hand side of equation (4) by $S$ yields the new inferred relative abundances. We note that a decrease (increase) in the calculated value of $S$ corresponds to a decrease (increase) in the inferred FIP enhancement for a given value of $R_{A 1} / R_{A 2}$.

\subsection{Effects}

For our calculations of $S$, we made a number of simplifying assumptions. For the pairs of lines we are concerned with here, to a first approximation the values of $C_{j} f_{q}$ have the same temperature dependence. To simplify the calculations, we have assumed a flat DEM $\left(\propto T^{0}\right)$. We further simplify the calculations by neglecting the temperature dependence of $C_{j}$, setting it equal to 1 for each ion. In this way we are able to focus specifically on how the uncertainty in the DR rate coefficients affects $S$. We then calculated $S$ for all 1728 sets of DR rate coefficients. The calculations were carried out over the temperature range $\Delta T$ from $\log T=5.0$ to 7.0 . This covers the temperature range over which the ions of interest form. For brevity, we have listed in Table 2 only the range of $S$ values for all 1728 variations. Clearly, the uncertainties in the DR rate coefficients can have a dramatic effect on any inferred FIP enhancement factors. We note that Table 2 does not display the corre-

TABLE 2

RANGe OF SCALE FACTOR $S$ FOR ALl 1728 SETS OF DR VARIATIONS (UNCONSTRAINED) AND FOR THE REDUCED SET OF

\begin{tabular}{|c|c|c|c|c|}
\hline \multirow[b]{2}{*}{ Line Ratio } & \multicolumn{2}{|c|}{ UNCONSTRAINED $S$} & \multicolumn{2}{|c|}{ CONSTRAINED $S$} \\
\hline & Minimum & Maximum & Minimum & Maximum \\
\hline $\mathrm{Mg}$ vI/ $\mathrm{Ne}$ vI .... & 0.60 & 1.11 & 0.62 & 1.11 \\
\hline $\mathrm{Mg}$ VII / $\mathrm{Ne}$ VII ... & 0.67 & 1.22 & 0.75 & 1.22 \\
\hline Mg IX / S Ix ...... & 0.33 & 1.29 & 0.41 & 1.29 \\
\hline $\mathrm{Mg}$ IX $/ \mathrm{S} \times \ldots \ldots$ & 0.51 & 1.64 & 1.00 & 1.64 \\
\hline $\mathrm{Si} \operatorname{IX} / \mathrm{S}$ IX ......... & 0.20 & 1.01 & 0.24 & 1.00 \\
\hline $\mathrm{Si} \mathrm{Ix} / \mathrm{S} \times \ldots \ldots$ & 0.36 & 1.14 & 0.43 & 1.14 \\
\hline $\mathrm{Si} \times / \mathrm{S} \times \ldots \ldots .$. & 0.43 & 1.60 & 0.71 & 1.60 \\
\hline
\end{tabular}
274 VARIATIONS (CONSTRAINED) lations between the values of $S$ for different line ratios for each set of $D R$ variations.

We have reduced the set of DR variations using our current hypothesis for the structure of the FIP effect. Mg VII and $\mathrm{Ne}$ VII are transition region temperature ions, which have been observed by Laming et al. (1999) from close to the solar limb out into the corona (where these ions are observed far away in temperature from where they peak in abundance). The inferred FIP effect is expected to be $\approx 4$ in the coronal observations and decrease as one moves to transition region temperatures closer to the solar limb. This expected behavior for the $\mathrm{Mg} / \mathrm{Ne}$ abundance ratio can be seen in Table 3, which is reproduced here from Laming et al. (1999). Line emission from $\mathrm{Mg}$ IX and S X is always dominated by coronal temperature plasmas, and the $\mathrm{Mg} \mathrm{IX} / \mathrm{S} \mathrm{x}$ ratio is expected to display an FIP factor of 4 at all positions listed in Table 3. Laming et al. (1999) measured an FIP factor of $\approx 3$. For our selection criteria, we take only those DR variations for which the FIP effect inferred using the $\mathrm{Mg}$ VII/ $\mathrm{Ne}$ VII line ratio varies by less than $\pm 25 \%$ and which also increase the FIP effect inferred using $\mathrm{Mg}$ IX/S x. The $25 \%$ is based on the estimated errors in the measured line ratios. We found 548 sets that met these constraints. This set was reduced to 274 by taking into account the fact that changes in the DR rate coefficient onto neon-like Si V had essentially no effect on the results. We have listed in Table 2 the range of $S$ for this reduced set of variations. Clearly, even in this reduced set, the uncertainties in the DR rate coefficients can still have a dramatic affect on many inferred FIP enhancement factors.

\subsection{Solar Off-Limb Observations: A Further Test}

At heights of $50^{\prime \prime}$ or more from the solar limb, the corona becomes essentially isothermal. As one moves away from the solar limb, the amount of cooler transition region plasma decreases quickly with height, while the hotter coronal gas diminishes much less rapidly and quickly becomes the dominant component. A striking illustration of this in the case of a solar equatorial streamer is given in Feldman et al. (1999). In Figure 3 of their paper they plot the variation of intensity with distance from the solar limb for various transitions from ions of Si vII-Si XII. All line intensities $I$ show the same slope $d(\log I) / d r$ and hence the same temperature, which from the slope evaluates to about $1.5 \times 10^{6} \mathrm{~K}$. Feldman et al. plot in their Figure 4 the loci of emission measures determined from these Si ions. Possible calibration uncertainties exist for the Si XII results since it is at the extreme short wavelength end of the SUMER second-order bandpass (Laming et al. 1997). Ignoring the Si XII loci, the temperature determined from the intersection of the remaining emission measure loci is

TABLE 3

\begin{tabular}{|c|c|c|c|c|}
\hline Line Ratio & Position $1^{\mathrm{a}}$ & Position 2 & Position 3 & Position 4 \\
\hline Mg VI $1190.09 / \mathrm{Ne}$ VI 558.59 . & & $7.4 \pm 1.4$ & $4.5 \pm 0.8$ & $3.5 \pm 0.8$ \\
\hline Mg VII $868.11 / \mathrm{Ne}$ VII $895.17 \ldots \ldots$. & $2.0 \pm 0.4$ & $2.6 \pm 0.5$ & $4.1 \pm 0.7$ & $4.1 \pm 0.7$ \\
\hline Mg Ix $749.55 /$ S Ix $871.71 \ldots \ldots \ldots$ & $3.6 \pm 0.8$ & $3.5 \pm 0.4$ & $3.2 \pm 0.1$ & $3.1 \pm 0.1$ \\
\hline Mg Ix $749.55 / \mathrm{S} \times 776.37 \ldots \ldots \ldots$ & $3.0 \pm 1.1$ & $3.2 \pm 1.0$ & $2.6 \pm 0.8$ & $3.0 \pm 0.9$ \\
\hline Si Ix $950.14 /$ S Ix $871.71 \ldots \ldots \ldots \ldots$ & $4.8 \pm 2.4$ & $3.6 \pm 0.5$ & $2.7 \pm 0.1$ & $2.8 \pm 0.1$ \\
\hline Si Ix $950.14 /$ S x $776.37 \ldots \ldots \ldots \ldots$ & $4.6 \pm 1.7$ & $3.0 \pm 0.9$ & $2.0 \pm 0.7$ & $2.4 \pm 0.8$ \\
\hline
\end{tabular}

ObSeRved Coronal FIP Fractionations (From Laming et AL. 1999)

a Position 1 corresponds to a slit position covering 14" in radial distance over the solar limb. Positions 2, 3, and 4 are successively 14" further out in radial distance from the solar limb. 


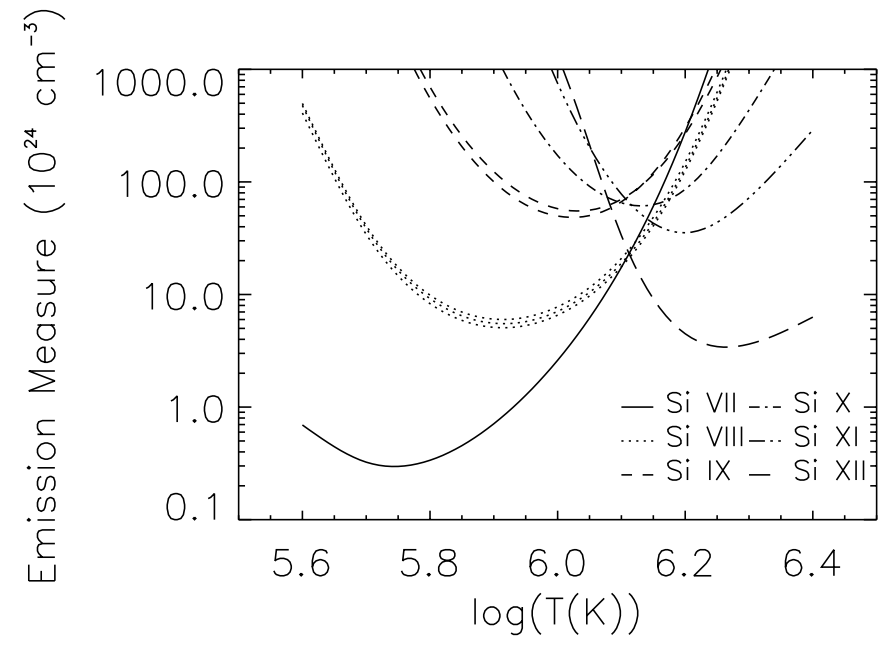

FIG. 2.-Emission measure loci for the ions Si VII-Si XII determined from SUMER observations of an equatorial streamer using the ionization balance calculations of Mazzotta et al. (1998). The curves intersect at a temperature close to $\log T=6.14$, suggesting that the conclusion that the plasma is isothermal. The spectral lines considered for each ion are $\mathrm{Si}$ VII $\lambda 1049.22$, Si VIII $\lambda 944.38, \lambda 949.22$, and $\lambda 1445.75$, Si Ix $\lambda 950.14$ and $\lambda 694.70$, Si x $\lambda 638.94$, Si XI $\lambda 580.91$, and Si XII $\lambda 499.41$. These give three curves for $\mathrm{Si}$ VIII, two for Si Ix, and one for each remaining ion.

$\log T=6.11 \pm 0.04$. This is similar to that determined from the height dependence of the emission in the various lines, assumed to fall off according to hydrostatic equilibrium.

Feldman et al. (1999) used the older ionization balance of Arnaud \& Rothenflug (1985). In order to assess the impact of our suggested changes to dielectronic recombination rate coefficients, we have remeasured the line intensities and replotted the emission measure determined using the ionization balance of Mazzotta et al. (1998). These results are shown in Figure 2. Comparing with Figure 4 of Feldman et al. (1999), we can see that the temperature at which the various curves intersect is now given by $\log T=6.14 \pm 0.05$.

We have investigated the effect of varying the DR rate coefficients in the $\mathrm{Si}$ ionization balance calculations. We have looked at variations of the rate coefficients in a "trial and error" fashion. Nine plausible sets of variations are given in Table 4. Others exist among our 1728 attempts, but these nine serve to illustrate one effect we are interested in. In Figure $3 a$ we replot the temperature region $6.0<\log T<6.3$ from the previous plot. Figures $3 b, 3 c$, and $3 d$ give the plots corresponding to variations 4,2 , and 3 , respectively, of the Mazzotta et al. (1998) DR rate coefficients. Variations $4,5,7$, and 9 are very similar, as are variations 1, 2, 6 and 8. The curves due to Si XII $\lambda 499.41$ have been omitted from these plots because of suspicions about the instrument calibration. In all panels the degree of overlap between the various intersections of the emission measure loci has improved. The improvement is best for variation 3, followed by variation 2 . Variation 4 is the least successful. Thus, the important conclusion that we are observing an isothermal plasma is, if anything, strengthened by our considerations of these plausible variations in the DR rate coefficients.

\section{CONCLUSIONS}

We have described on the basis of atomic physics theory what uncertainties may be present in the DR rate coefficients commonly used in ionization balance calculations. Of course, this situation will only be definitely improved by further calculations and experiments, but given the number of individual rate coefficients required for astrophysical modeling purposes, this is an enormous amount of work. To help prioritize the needed atomic data and to provide further motivation, we have investigated how the uncertainties in the DR rate coefficients translate into uncertainties in inferred FIP factors. We find that, depending on the specific line ratio, inferred FIP factors can be a factor of 5 smaller or 1.6 times larger than the FIP factor inferred using the unscaled DR rate coefficients of Mazzotta et al. (1998).

Taking the unconstrained data in Table 2 and using the ratio of the maximum over minimum value of $S$ for each line ratio, we can prioritize the need for DR measurements and calculations of the various isoelectronic sequences studied here. Listing the various line ratios in decreasing order of $S_{\max } / S_{\min }$ yields $\mathrm{Si}$ IX/S IX, Mg IX/S Ix, Si x/S x, Mg IX/S x, Si IX/S X, Mg VI/Ne VI, and $\mathrm{Mg}$ VII/Ne VII. Based on the order and frequency with which the different isoelectronic sequences occur in this list, we prioritize our list of needed rate coefficients for DR onto specific isoelectronic sequences, in decreasing order of importance, as follows: O-like, C-like, Be-like, N-like, and B-like. To this we append F-like, Li-like, He-like, and Ne-like based on the range of the DR scale factors given in Table 4.

We have arrived at our list through admittedly subjective means, but we believe that in this case the ends justify the

TABLE 4

Scale Factors for Initial Ions by Which the Recommended DR Rate CoefFicients of Mazzotta et al. (1998) Have BeEn Multiplied by for the Nine Variations Selected in a “Trial and Error” Fashion

\begin{tabular}{lccccccccc}
\hline & \multicolumn{8}{c}{ ISOELECTRONIC SEQUENCE } \\
\cline { 2 - 9 } VARIATION NUMBER & $\mathrm{He}$ & $\mathrm{Li}$ & $\mathrm{Be}$ & $\mathrm{B}$ & $\mathrm{C}$ & $\mathrm{N}$ & $\mathrm{O}$ & $\mathrm{F}$ & $\mathrm{Ne}$ \\
\hline $1 \ldots \ldots \ldots \ldots \ldots \ldots \ldots$ & 1.0 & 0.8 & 1.0 & 1.7 & 0.62 & 1.0 & 1.0 & 1.0 & 1.0 \\
$2 \ldots \ldots \ldots \ldots \ldots \ldots \ldots$ & 1.0 & 0.8 & 1.0 & 1.7 & 0.62 & 1.0 & 1.0 & 4.7 & 1.0 \\
$3 \ldots \ldots \ldots \ldots \ldots \ldots \ldots$ & 1.0 & 0.8 & 1.6 & 1.7 & 0.62 & 1.0 & 1.0 & 4.7 & 1.0 \\
$4 \ldots \ldots \ldots \ldots \ldots \ldots \ldots$ & 1.0 & 1.0 & 1.0 & 1.0 & 0.62 & 1.0 & 1.0 & 1.0 & 1.0 \\
$5 \ldots \ldots \ldots \ldots \ldots \ldots \ldots$ & 1.0 & 1.0 & 1.0 & 1.0 & 0.62 & 1.0 & 1.0 & 4.7 & 1.0 \\
$6 \ldots \ldots \ldots \ldots \ldots \ldots \ldots$ & 1.2 & 0.8 & 1.0 & 1.7 & 0.62 & 1.0 & 1.0 & 1.0 & 1.0 \\
$7 \ldots \ldots \ldots \ldots \ldots \ldots \ldots$ & 1.2 & 1.0 & 1.0 & 1.0 & 0.62 & 1.0 & 1.0 & 1.0 & 1.0 \\
$8 \ldots \ldots \ldots \ldots \ldots \ldots \ldots$ & 1.2 & 1.0 & 1.0 & 1.7 & 0.62 & 1.0 & 1.0 & 1.0 & 1.0 \\
$9 \ldots \ldots \ldots \ldots \ldots \ldots \ldots$ & 1.2 & 1.2 & 1.0 & 1.0 & 0.62 & 1.0 & 1.0 & 1.0 & 1.0 \\
\hline
\end{tabular}



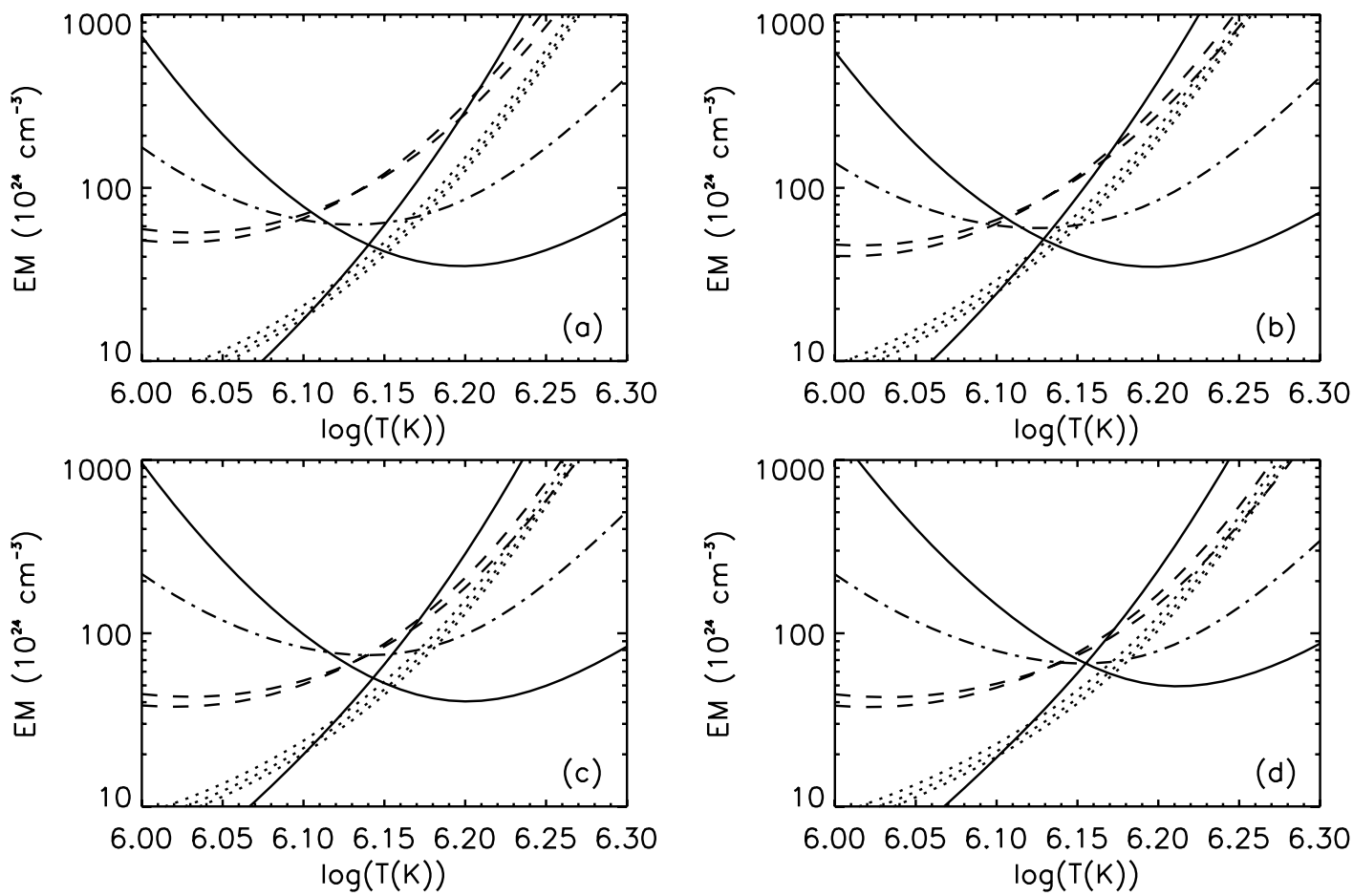

Fig. 3.-Emission measure loci from Fig. 2 for the temperature region $6.0 \leq \log T \leq 6.3$. (a) Results using the Mazzotta et al. (1998) ionization balance. (b) $-(d)$ Plots corresponding to variations 4, 2, and 3, respectively, of the Mazzotta et al. ionization balance. Variations 4, 5, 7, and 9 are very similar, as are variations 1, 2,6 and 8. As can be seen from the figure, variation 3 appears to give the best improvement to the inferred (and expected) isothermal nature of the observed coronal plasma, followed by variation 2 , with variation 4 being the least successful. The curves due to Si XII $\lambda 499.41$ have been omitted from these plots because of suspicions about the instrument calibration.

means. Faced with the current degree of uncertainty in the dielectronic recombination rate coefficients relevant to astrophysical plasmas, the number of required calculations and measurements is daunting. Our aim in this work has been to prioritize this work and to point out those ions where the uncertainties in the DR rate coefficients have the most impact on the analysis of astrophysical UV and X-ray spectra.

We thank Arati Dasgupta, Verne Jacobs, Pasquale Mazzotta, Kengo Moribayashi, and Takako Kato for stimu- lating conversations and correspondence. D. W. S. was supported in part by NASA Solar Physics Research, Analysis, and Suborbital Program grant NAG 5-9581 to Columbia University. J. M. L. was supported by the NRL/ONR Solar Magnetism and the Earth's Environment 6.1 Research Option and by NASA Contracts W19473, W19539, and the 2000 SEC Guest Investigator Program. The SUMER project is financially supported by DARA, CNES, NASA, and the ESA PRODEX program (Swiss Contribution). SUMER is a part of $S O H O$, the Solar and Heliospheric Observatory, of ESA and NASA.

\section{REFERENCES}

Aldrovandi, S. M. V., \& Péquignot, D. 1973, A\&A, 25, 137 (erratum 47, 321 [1976])

Ali, R., Bhalla, C. P., Cocke, C. L., Schulz, M., \& Stockli, M. 1991, Phys. Rev. A, 44, 223

Andersen, L. H., Bolko, J., \& Kvistgaard, P. 1990, Phys. Rev. A, 41, 1293

Andersen, L. H., Pan, G.-Y., Schmidt, H. T., Pindzola, M. S., \& Badnell,

N. R. 1992, Phys. Rev. A, 45, 6332

Arnaud, M., \& Raymond, J. C. 1992, ApJ, 398, 394

Arnaud, M., \& Rothenflug, R. 1985, A\&AS, 60, 425

Badnell, N. R. 1986, J. Phys. B, 19, 3827

. 1987, J. Phys. B, 20, 2081

1988, J. Phys. B, 21, 749

1992, Phys. Rev. A, 46, 660

Badnell, N. R., \& Pindzola, M. S. 1989a, Phys. Rev. A, 39, 1685

1989b, Phys. Rev. A, 39, 1690

Badnell, N. R., Pindzola, M. S., Andersen, L. H., Bolko, J., \& Schmidt, H. T. 1991, J. Phys. B, 24, 4441

Bartsch, T., et al. 1997, Phys. Rev. Lett., 79, 2233

Beiersdorfer, P., Phillips, T. W., Wong, K. L., Marrs, R. E., \& Vogel, D. A. 1992, Phys. Rev. A, 46, 3812

Belić, D. S., \& Pradhan, A. K. 1987, Comments At. Mol. Phys., 20, 317

Bell, R. H., \& Seaton, M. J. 1985, J. Phys. B, 18, 1589

Brinkmann, A. C., et al. 2001, A\&A, 365, L324

Burgess, A. 1965, ApJ, 141, 1588

Burgess, A., \& Tworkowski, A. S. 1976, ApJ, 205, L105

Cargill, P. J. 1994, ApJ, 422, 381
Cargill, P. J., \& Klimchuk, J. A. 1997, ApJ, 478, 799

Chen, M. H. 1985, Phys. Rev. A, 31, 1449

.1986, Phys. Rev. A, 33, 994

1988a, Phys. Rev. A, 38, 2332

. 1991, Phys. Rev. A, 44, 4215

Cowan, R. D. 1981, The Theory of Atomic Structure and Spectra (Berkeley: Univ. California Press)

Culhane, J. L., Korendyke, C. M., Watanabe, T., \& Doschek, G. A. 2002, Proc. SPIE, in press

Dasgupta, A., \& Whitney, K. G. 1990, Phys. Rev. A, 42, 2640

. 1994, At. Data Nucl. Data Tables, 58, 77

del Zanna, G., \& Bromage, B. J. I. 1999, J. Geophys. Res., 104, 9753

DeWitt, D. R., Lindroth, E., Schuch, R., Gao, H., Quinteros, T., \& Zong, W. 1995, J. Phys. B, 28, L147

DeWitt, D. R., Schuch, R., Gao, H., Zong, W., Asp, S., Biedermann, C., Chen, M., H., \& Badnell, N. R. 1996, Phys. Rev. A, 53, 2327

Dittner, P. F., Datz, S., Miller, P. D., Pepmiller, P. L., \& Fou, C. M. 1987, Phys. Rev. A, 35, 3668

Doschek, G. A., \& Laming, J. M. 2000, ApJ, 539, L71

Doschek, G. A., Laming, J. M., Feldman, U., Wilhelm, K., Lemaire, P., Schühle, U., \& Hassler, D. M. 1998, ApJ, 504, 573

Drake, J. J., Brickhouse, N. S., Kashyap, V., Laming, J. M., Huenemoerder, D. P., Smith, R., \& Wargelin, B. J. 2001, ApJ, 548, L81

Drake, J. J., Laming, J. M., \& Widing, K. G. 1997, ApJ, 478, 403

Drake, J. J., Laming, J. M., Widing, K. G., Schmitt, J. H. M. M., Haisch, B., \& Bowyer, S. 1995, Science, 267, 1470 
Feldman, U., Doschek, G. A., Schühle, U., \& Wilhelm, K. 1999, ApJ, 518, 500

Feldman, U., \& Laming, J. M. 1994, ApJ, 434, 370 2000, Phys. Scr., 61, 222

Feldman, U., \& Widing, K. G. 1990, ApJ, 363, 292

.1993, ApJ, 414, 381

Fournier, K. B., Cohen, M., \& Goldstein, W. H. 1997, Phys. Rev. A, 56,

Gorczyca, T. W., \& Badnell, N. R. 1996, Phys. Rev. A, 54, 4113

Griem, H. R. 1988, J. Quant. Spectrosc. Radiat. Transfer, 40, 403

Griffin, D. C., Pindzola, M. S., \& Bottcher, C. 1985, Phys. Rev. A, 31, 568

Gu, M. F. 2000, Ph.D. thesis, Columbia Univ.

Gu, M. F., et al. 1999, ApJ, 518, 1002

Hahn, Y. 1993, J. Quant. Spectrosc. Radiat. Transfer, 49, 81

Harrison, R. A., et al. 1997, Sol. Phys., 170, 123 1995, Sol. Phys., 162, 233

Jacobs, V. L., Davis, J., Keeple, P. C., \& Blaha, M. 1977a, ApJ, 211, 605 $1977 \mathrm{~b}$, ApJ, 215, 690

Jacobs, V. L., Davis, J., \& Rogerson, J. E. 1978, J. Quant. Spectrosc. Radiat. Transfer, 19, 591

Jacobs, V. L., Davis, J., Rogerson, J. E., \& Blaha, M. 1979, ApJ, 230, 627

Jordan, C. 1980, A\&A, 86, 355

Karim, K. R., \& Bhalla, C. P. 1989, Phys. Rev. A, 39, 3548

Kenntner, J. 1995, Ph.D. thesis, Univ. Heidelberg

Kenntner, J., et al. 1995, Nucl. Instrum. Methods Phys. Res., B98, 142

Laming, J. M., Drake, J. J., \& Widing, K. G. 1995, ApJ, 443, 416

Laming, J. M., Feldman, U., Drake, J. J., \& Lemaire, P. 1999, ApJ, 518, 926

Laming, J. M., Feldman, U., Schühle, U., Lemaire, P., Curdt, W., \& Wilhelm, K. 1997, ApJ, 485, 911

Landini, M., \& Monsignori Fossi, B. C. 1991, A\&AS, 91, 183

Lemaire, P., et al. 1997, Sol. Phys., 170, 105

Linkemann, J. et al. 1995, Nucl. Instrum. Methods Phys. Res., B98, 154

Malinovsky, M., \& Heroux, L. 1973, ApJ, 181, 1009

Mannervik, S., DeWitt, D., Engström, L., Lidberg, J., Lindroth, E., Schuch, R., \& Zong, W. 1998, Phys. Rev. Lett., 81, 313

Mazzotta, P., Mazzitelli, G., Colafrancesco, S., \& Vittorio, N. 1998, A\&AS, 133,403

McLaughlin, D. J., \& Hahn, Y. 1984, Phys. Rev. A, 29, 712

Merts, A. L., Cowan, R. D., \& Magee, Jr., N. H. 1976, Los Alamos Scientific Laboratory Report LA-6220-MS (March 1976)

Mitnik, D. M., et al. 1998, Phys. Rev. A, 57, 4365

Müller, A. 1995, in Atomic and Plasma-Material Interaction Data for Fusion (Nucl. Fusion Suppl, Vol. 6; Vienna : IAEA), 59

Müller, A., Belić, D. S., DePaola, B. D., Djurić, N., Dunn, G. H., Mueller, D. W., \& Timmer, C. 1987, Phys. Rev. A, 36, 599

Nahar, S. N. 1995, ApJS, 101, 423 (erratum 106, 213 [1996]) 1999, ApJS, 120, 131

Nahar, S. N., \& Pradhan, A. K. 1994, Phys. Rev. A, 49, 1816 . 1997, ApJS, 111, 339

Nilsen, J. 1986, J. Phys. B, 19, 2401

Noci, G., Spadaro, D., Zappala, R. A., \& Zuccarello, F. 1988, A\&A, 198, 311

Nussbaumer, H., \& Storey, P. J. 1983, A\&A, 126, 75 1984, A\&AS, 56, 293
Nussbaumer, H., \& Storey, P. J. 1986, A\&AS, 64, 545 1987, A\&AS, 69, 123

Press, W. H., Teukolsky, S. A., Vetterling, W. T., \& Flannery, B. P. 1992, Numerical Recipes (2d Ed.; Cambridge: Cambridge Univ. Press)

Ramadan, H. H., \& Hahn, Y. 1989, Phys. Rev. A, 39, 3350

Robicheaux, F., Gorczyca, T. W., Pindzola, M. S., \& Badnell, N. R. 1995 , Phys. Rev. A, 52, 1319

Romanik, C. J. 1988, ApJ, 330, 1022

Roszman, L. J. 1987a, Phys. Rev. A, 35, 2122

. 1987b, Phys. Rev. A, 35, 2138

. 1987c, Phys. Rev. A, 35, 3368

1989, Phys. Scr., T28, 36

Safronova, U. I., \& Kato, T. 1998, J. Phys. B, 31, 2501

Savin, D. W. 1999, ApJ, 523, 855 2000, ApJ, 533, 106

Savin, D. W., Gardner, L. D., Reisenfeld, D. B., Young, A. R., \& Kohl, J. L. 1996, Phys. Rev. A, 53, 280

Savin, D. W., et al. 1997, ApJ, 489, L115 1999, ApJS, 123, 687

2000, in Proceedings of the 12th APS Topical Conference on Atomic Processes in Plasmas, Reno Nevada, ed. R. C. Mancini (New York: American Institute of Physics), 267

Schennach, S., et al. 1994, Z. Phys. D, 30, 291

Schippers, S., Batsch, T., Brandau, C., Gwinner, G., Linkemann, J., Müller, A., Saghiri, A. A., \& Wolf, A. 1998, J. Phys. B, 31, 4873

Schippers, S., Müller, A., Gwinner, G., Linkemann, J., Saghiri, A. A., \& Wolf, A. 2001, ApJ, 555, 1027

Shull, J. M., \& van Steenberg, M. 1982, ApJS, 48, 95 (erratum 49, 351)

Smith, A. J., Beiersdorfer, P., Decaux, V., Widmann, K., Reed, K. J., \& Chen, M. H. 1996, Phys. Rev. A, 54, 462

Smith, A. J., Beiersdorfer, P., Widmann, K., Chen, M. H., \& Scofield, J. H. 2000, Phys. Rev. A, 62, 052717

Terao, M., Bell, K. L., Burke, P. G., \& Hibbert, A. 1991, J. Phys. B, 24, L 321

von Steiger, R., Geiss, J., \& Gloeckler, G. 1997, in Cosmic Winds and the Heliosphere, ed. J. R. Jokopii, C. P. Sonett, \& M. S. Giampapa (Tucson Univ. Arizona Press), 581

Wargelin, B. J., Kahn, S. M., \& Beiersdorfer, P. 2001, Phys. Rev. A, 63, 022710

White, S. M., Thomas, R. J., Brosius, J. W., \& Kundu, M. R. 2000, ApJ, 534, L203

Widing, K. G., \& Feldman, U. 1989, ApJ, 344, 1046 1992, ApJ, 392, 715

.1993, ApJ, 416, 392

Wilhelm, K., et al. 1995, Sol. Phys., 162, 189

. 1997, Sol. Phys., 170, 75

Wolf, A. 1999, in Atomic Physics with Heavy Ions, ed. H. F. Beyer \& V. P. Shevelko, (Berlin: Springer-Verlag), 3

Young, P. R., Klimchuk, J. A., \& Mason, H. E. 1999, A\&A, 350, 286

Young, P. R., \& Mason, H. E. 1997, Sol. Phys., 175, 523

Zong, W., Schuch, R., Gao, H., DeWitt, D. R., \& Badnell, N. R. 1998, J. Phys. B, 31, 3729

Zong, W., Schuch, R., Lindroth, E., Gao, H., DeWitt, D. R., \& Asp, S. 1997, Phys. Rev. A, 56, 386 